\title{
The marvelous optical performance of AIGaN-based deep ultraviolet light-emitting diodes with AllnGaN-based last quantum barrier and step electron blocking layer
}

\author{
Tariq Jamil $^{1} \cdot$ Muhammad Usman $^{1}$ (1) $\cdot$ Shahzeb Malik ${ }^{1} \cdot$ Habibullah Jamal $^{1}$ \\ Received: 3 February 2021 / Accepted: 28 April 2021 / Published online: 4 May 2021 \\ (C) The Author(s), under exclusive licence to Springer-Verlag GmbH, DE part of Springer Nature 2021
}

\begin{abstract}
The optoelectronic characteristics of AlGaN-based deep ultraviolet light-emitting diodes (DUV LEDs) with quaternary last quantum barrier (QLQB) and step-graded electron blocking layer (EBL) are investigated numerically. The results show that the internal quantum efficiency (IQE) and radiative recombination rate are remarkably improved with AlInGaN step-graded EBL and QLQB as compared to conventional or ternary AlGaN EBL and last quantum barrier (LQB). This significant improvement is assigned to the optimal recombination of electron-hole pairs in the multiple quantum wells (MQWs). It is due to the decrease in strain and lattice mismatch between the epi-layers which alleviates the effective potential barrier height of the conduction band and suppressed the electron leakage without affecting the holes transportation to the active region. Moreover, to figure out quantitatively, the electron and hole quantity increased by $\sim 25 \%$ and $\sim 15 \%$, respectively. Additionally, the IQE and radiative recombination rate are enhanced by $48 \%$ and 55\%, respectively, as compared to conventional LED. So, we believe that our proposed structure is not only a feasible approach for achieving highly efficient DUV LEDs, but the device physics presented in this study establishes a fruitful understanding of III nitride-based optoelectronic devices.
\end{abstract}

Keywords DUV LEDs · AlInGaN · Efficiency $\cdot$ Optoelectronic performance

\section{Introduction}

The real-world applications, such as water and air disinfection, currency screening, decontamination, photolithography, color display, and even for the disinfection of the SARS$\mathrm{CoV}-2$ virus, require a narrow UV band spectrum [1-5]. AlGaN-based semiconductor alloys have unique characteristics which make them a promising candidate for the above applications $[6,7]$. Due to these remarkable characteristics, the AlGaN-based UV LEDs have been keenly studied in the last two decades $[8,9]$. Despite the extensive research, the optoelectronic performance of DUV LEDs is still considerably low. This poor performance of AlGaN-based UV LEDs is due to many factors $[8,10]$. High threading dislocation density (TDD) and Auger recombination also severely affect

Muhammad Usman

m.usman@giki.edu.pk

Faculty of Engineering Sciences, Ghulam Ishaq Khan Institute of Engineering Sciences and Technology, Topi 23460, Khyber Pakhtunkhwa, Pakistan the optoelectronic performance of DUV LEDs. Researchers proposed different DUV structures to enhance the optical characteristics and reducing highly TDD using buffer layers or patterned sapphire substrates $[9,11]$, p-doped deltaaccelerating [12], and quaternary-graded quantum barrier [13]. It is worth noting that electrons have lower effective thermal velocity due to their higher effective mass, so electrons easily overcome the effective potential barrier height (denoted by $\Phi_{\mathrm{e}}$ ) of quantum barriers and EBL, resulting in leakage to the p-type layers [14]. Conversely, the holes have lower thermal velocity due to their higher effective mass. For holes, it is difficult to overcome the effective potential barrier height (denoted by $\Phi_{h}$ ) of EBL and quantum barriers. Eventually, the leaked electrons recombine with holes in the p-type layer, leading to non-radiative recombination instead of radiative recombination $[14,15,16]$. Through this, the uneven distribution of carriers occurs, which further stymies the AlGaN-based DUV LEDs performance [17]. To tackle this issue, many sophisticated approaches have been used such as V-shaped EBL [18], W-shaped EBL [19], irregular sawtooth EBL [20], trapezoidal EBL [21], and anti-trapezoidal EBL [22]. 
Keeping this in mind, we have proposed QLQB and quaternary step-graded EBL and study their effect on the device output. Previously, our group studied the effect of QLQB as well as EBL simultaneously on the carrier leakage [13]. Based on simulation results, it was found that the electron and hole concentrations are remarkably enhanced in our proposed DUV LED. Although our study is theoretical, we expect that experimental implementation is quite simple because many groups fabricated the AlInGaN-based highly efficient layer for DUV LEDs, through many epitaxial technologies such as molecular beam epitaxy (MBE) and metal-organic chemical vapor deposition (MOCVD) [23, 24]. To the best of our knowledge, the effect of QLQB and quaternary step-graded EBL on DUV LEDs has not been reported to date.

\section{Device structure and parameters}

The AlGaN-based DUV LED denoted as (LED-A) is used as a reference structure in this study. The reference structure (LED A) comprises of a $3-\mu \mathrm{m}$-wide electron injector $\mathrm{n}-\mathrm{Al}_{0.6} \mathrm{Ga}_{0.4} \mathrm{~N}$ layer with doping concentration (Si-doped) of $1 \times 10^{18} \mathrm{~cm}^{-3}$, the active region contains five un-doped $\mathrm{Al}_{0.45} \mathrm{Ga}_{0.55} \mathrm{~N}(2 \mathrm{~nm}) \mathrm{QW}$ and six un-doped $\mathrm{Al}_{0.56} \mathrm{Ga}_{0.44} \mathrm{~N}$ (12 nm) QB, followed by a 10-nm-thick $\mathrm{Al}_{0.6} \mathrm{Ga}_{0.4} \mathrm{~N}$ EBL layer with doping concentration (Mg-doped) of $3 \times$ $10^{19} \mathrm{~cm}^{-3}$ is used. The hole injector layers, i.e., $\mathrm{Al}_{0.4} \mathrm{Ga}_{0.6} \mathrm{~N}$ have a thickness of $50 \mathrm{~nm}$ and a doping concentration of $3 \times 10^{19} \mathrm{~cm}^{-3}$ followed by a 50 -nm-wide p-contact layer $\mathrm{p}-\mathrm{GaN}$ with a doping concentration of $3 \times 10^{19} \mathrm{~cm}^{-3}$. The proposed LED denoted as (LED B) is identical to LED A except that the conventional EBL is divided into five layers of $\mathrm{Al}_{\mathrm{x}} \mathrm{In}_{\mathrm{y}} \mathrm{Ga}_{\mathrm{z}} \mathrm{N}$, each has a thickness of $2 \mathrm{~nm}$. Further, the EBL is step-graded such that the composition value $x, y$, $\mathrm{z}$ for the first EBL is $(0.7,0.02,0.28)$, followed by a $10 \%$ decrease of Aluminum (Al) composition in the next layer $(0.6,0.02,0.38)$ and so on up to five consecutive EBLs and in the last layer composition $(\mathrm{x}, \mathrm{y}, \mathrm{z})$ of EBL is $(0.3$, $0.02,0.68)$. Change in composition of $\mathrm{GaN}$ is related to $\mathrm{Al}$ content. Additionally, in the proposed LED, AlInGaN LQB $\left(\mathrm{Al}_{0.56} \mathrm{In}_{0.02} \mathrm{Ga}_{0.42} \mathrm{~N}\right)$ layer is also introduced at the same time instead of ternary $\mathrm{Al}_{0.56} \mathrm{Ga}_{0.44} \mathrm{~N}$. The schematic of LED A is shown in Fig. 1a, while the energy band of LEDs is sketched in Fig. 1b. In Fig. 1b, the black and red lines represent the conduction band of LED A and LED B, respectively. As shown in Fig. $1 b$ the $\mathrm{Al}$ content in EBL is constant and the width of EBL is $10 \mathrm{~nm}$ in LED A. In LED B, the $\mathrm{Al}$ content is decreased step-wise by $10 \%$ in every layer and the $10 \mathrm{~nm}$ EBL is divided into five layers (each $2 \mathrm{~nm}$ thick). It is worth noting that the LQB of conventional LED $(\mathrm{AlGaN})$ is replaced by $\mathrm{AlInGaN}$ in the proposed LED.

The LED structures have been numerically simulated using SiLENSe ${ }^{\text {TM }}$ 5.14. SiLENSe ${ }^{\text {TM }}$ is based on the 1D drift-diffusion model by considering some specific properties like threading dislocation density, low efficiency of the wurtzite materials, and strong piezoelectric polarization. The electron and hole fluxes calculated using a selfconsistent (SC) solution of the drift-diffusion transport and Poisson equations. The carrier wave functions are determined by solving the Schrödinger wave equation. The main characteristics of the SiLENSe tool are energy band profile, carrier concentration, radiative recombination rate, emission spectrum, carrier flux, and IQE. IQE is computed as [25].

$\eta_{I Q E}=\eta_{i n j} \cdot \frac{R_{\text {rad }}}{R_{\text {rad }}+R_{S R H}+R_{\text {Aug }}}$

where $\eta_{I Q E}$ is the IQE, $\eta_{i n j}$ is the injection quantum efficiency, $R_{S R H}$ is the SRH recombination radiative, $R_{\text {rad }}$ is

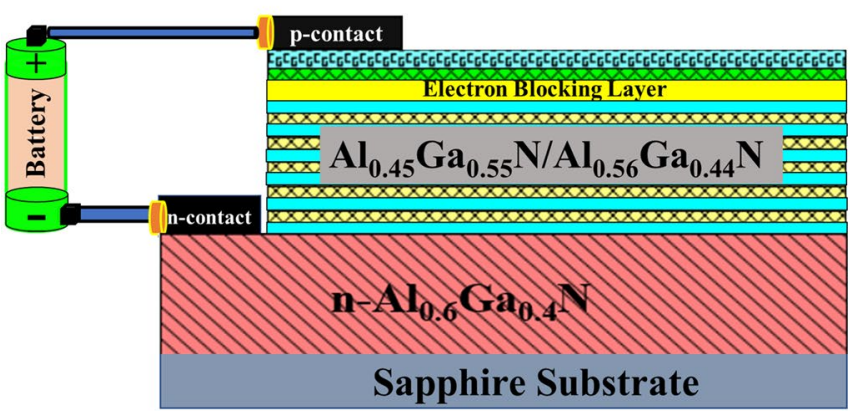

(a)

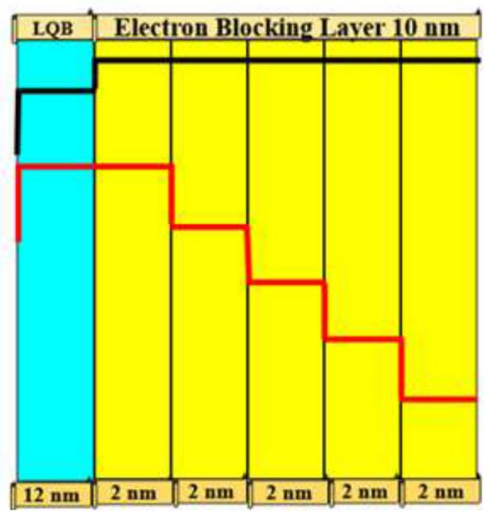

(b)

Fig. 1 Schematic of a LED A b Energy band rectangular LQB, step-graded EBL of both structures 
recombination rate, while $R_{\text {Aug }}$ is the Auger recombination rate. The defects that contribute to SRH recombination rate are computed as [26].

$R_{S R H}=\left(\frac{\tau_{n}^{t o t}}{n}+\frac{\tau_{p}^{t o t}}{p}\right)^{-1} \cdot\left[1-\exp \left(-\frac{F_{n}-F_{p}}{k T}\right)\right]$

where, the $\tau_{n}^{\text {tot }}, \tau_{p}^{\text {tot }}$ shows the electrons and holes lifetime, and $n, p, F_{n}$, and $F_{p}$ are the electron and hole concentration at quasi-Fermi level, respectively. The non-radiative lifetime is calculated by [25].

$\tau_{n, p}^{t o t}=\left(\frac{1}{\tau_{n, p}^{d e f}}+\frac{f_{n, p}}{\tau_{n, p}^{d i s}}\right)^{-1}$

where, $\tau_{n, p}^{d i s}$ is non-radiative lifetime due to some defects such as impurities and vacancies. Similarly, the relation for the total recombination rate inside the LED QWs is written as

$R=A n+B n^{2}+C n^{3}$

where $n, A, B, C$ are the carrier concentration, SRH recombination coefficient, radiative recombination coefficient, and Auger recombination coefficient.

The overall area of the LED chips are $300 \mu \mathrm{m} \times 300 \mu \mathrm{m}$. Other parameters used in this work are the band offset ratio of conduction and valence band, i.e., 0.7/0.3, Auger Coefficient, i.e., $2.5 \times 10^{-30} \mathrm{~cm}^{6} \mathrm{~s}^{-1}$. Shockley-Read-Hall (SRH) lifetime, i.e., $10 \mathrm{~ns}$ [27]. Electron and hole mobility of 100 $\mathrm{cm}^{2} / \mathrm{V}$.s and $10 \mathrm{~cm}^{2} / \mathrm{V}$.s, respectively, is employed while the operating temperature is set at $300 \mathrm{~K}$. Some important parameters used in simulations are listed in Table 1. The remaining parameters of the materials and complete explanation of the simulation model are given elsewhere[27-29].

\section{Results and discussion}

Figure 2 presents the IQE profile for both LEDs. The peak IQE of LED A and LED B is $\sim 37 \%$ and $\sim 54 \%$, respectively. The IQE of LED B is increased by $48 \%$ as compared to LED A. Interestingly, the efficiency droop is severely decreased up to $~ 3 \%$ in LED B, while LED A exhibits $~ 52 \%$ droop at $200 \mathrm{~A} / \mathrm{cm}^{2}$. The amelioration in IQE and decline in efficiency droop are quite clear in our proposed structure. So, it

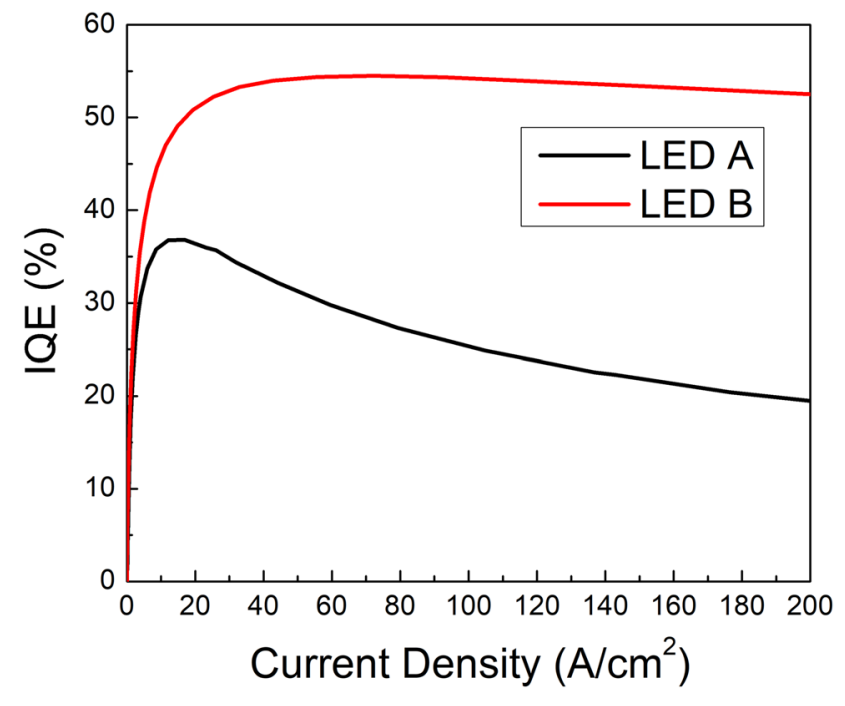

Fig. 2 Internal quantum efficiency as a function of current density

is evident that the optical characteristics of LED B (proposed structure) are improved markedly as compared to LED A.

Figure 3 illustrates quantitatively the number of electrons and holes at $80 \mathrm{~A} / \mathrm{cm}^{2}$. The electron concentration in a region of LED B is increased by $25 \%$ as compared to LED A as shown in Fig. 3a. It is worth noting that in the last quantum well the electron large concentration shows the high capability of electron confinement. Likewise, the holes in the MQWs for LED B are increased by $15 \%$ as compared to LED A, as shown in Fig. 3b. Thus, these results show that the LED B confine carriers in the active region effectively.

The enhancement in the carriers' concentrations in the MQWs leads to a decrease in the electron leakage and encourages the hole transport into MQWs. To verify this, the electron and hole current density as a function of position are plotted in Fig. 4a and b, respectively. The electron current density, for LED B, in p-type layers is lesser than LED A due to increased $\Phi$ e [30]. Conversely, the hole transport for LED B is 4.3 times greater than LED A as shown in Fig. 4b. Thus, the number of electrons is remarkably improved in the proposed structure as previously mentioned. It is principally because of the lower band-bending at the LQB and EBL interface due to lattice match, which leads to less carrier leakage in the proposed LED.
Table. 1 Important Materials Parameters used in Simulations Package [27, 28]

\begin{tabular}{lllccccc}
\hline SN & Parameter & Symbol (unit) & GaN & AlN & InN & $\begin{array}{c}\text { Bowing fac- } \\
\text { tor of InGaN } \begin{array}{l}\text { Bowing } \\
\text { factor of } \\
\text { AlGaN }\end{array}\end{array}$ \\
\hline 1 & Lattice constant & $\mathrm{a}(\AA)$ & 3.189 & 3.112 & 3.545 & - & - \\
2 & Band gap Energy $(300 K)$ & $\mathrm{E}_{\mathrm{g}}(\mathrm{eV})$ & 3.42 & 3.68 & 0.77 & 1.8 & 1.0 \\
3 & Spontaneous Polarization & $\mathrm{P}_{\mathrm{sp}}\left(\mathrm{C} / \mathrm{m}^{2}\right)$ & -0.034 & -0.090 & -0.042 & -0.037 & -0.021 \\
\hline
\end{tabular}




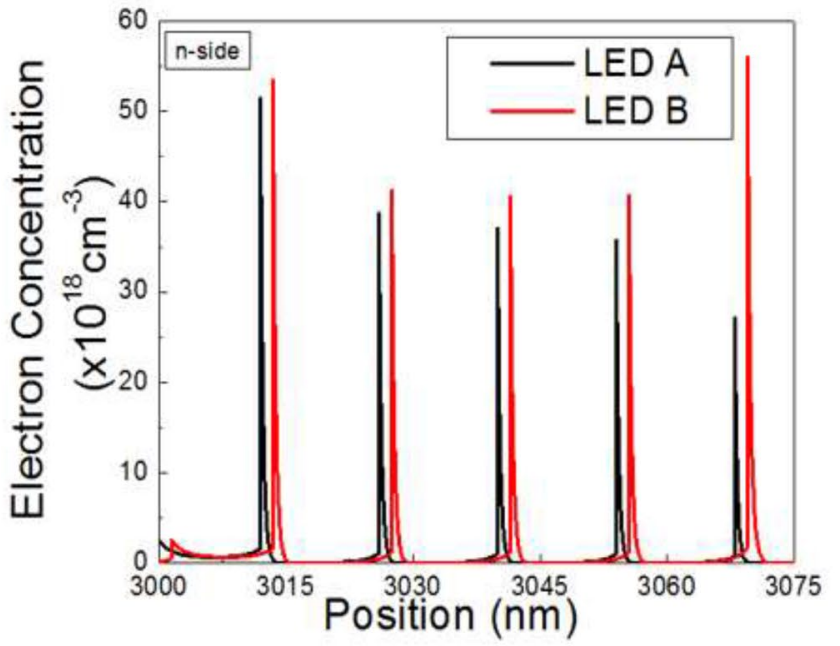

(a)

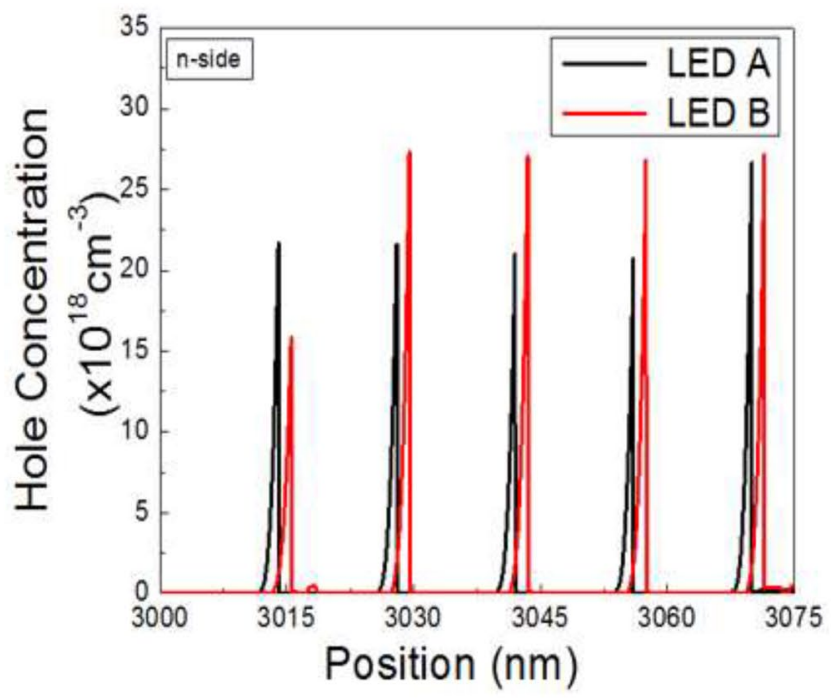

(b)

Fig. 3 Carriers' concentration in MQWs a Electron, b hole at $80 \mathrm{~A} / \mathrm{cm}^{2}$

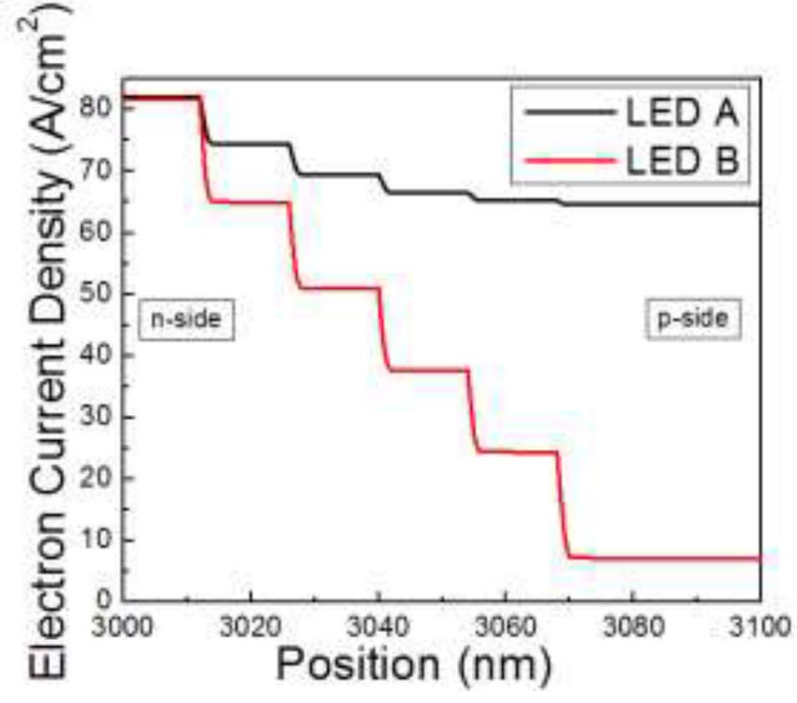

(a)

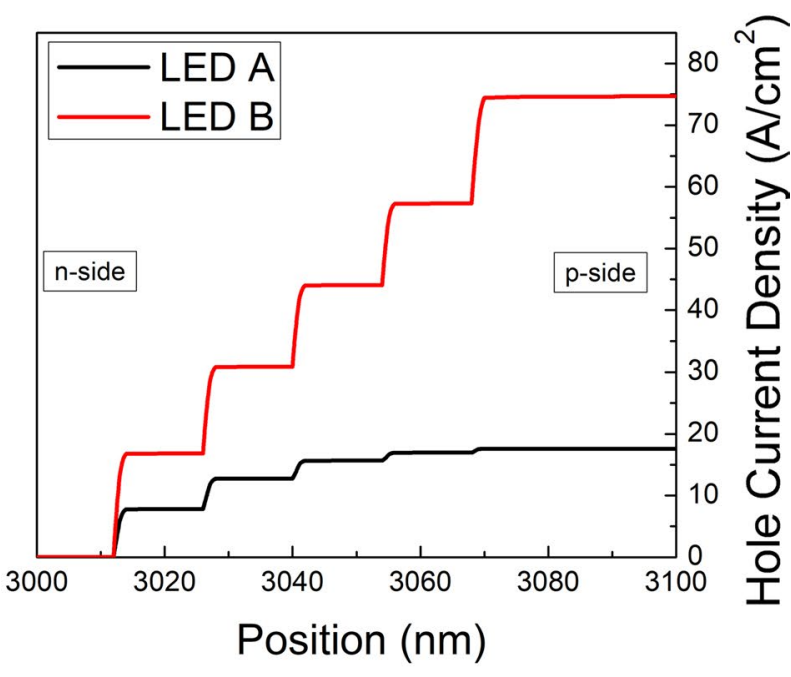

(b)

Fig. 4 a [Electron Current Density], b [Hole Current Density] in both LEDs at $80 \mathrm{~A} / \mathrm{cm}^{2}$

To explore the further reason for the amelioration of IQE in the proposed LED, the radiative recombination rate of both LEDs is investigated. Figure 5 shows the radiative recombination rate at $80 \mathrm{~A} / \mathrm{cm}^{2}$. The electron-hole pairs radiative recombination for LED B is enormously increased by $55 \%$ as compared to LED A. So, the elevation in radiative recombination is assigned to more electron-hole pairs confinement in the active zone which is explained above.
Magnified radiative recombination rate leads to the high emission intensity of DUV LED. Figure 6 presents the emission spectra of LEDs at the current density of $80 \mathrm{~A} / \mathrm{cm}^{2}$. The peak intensity of LED B is increased as compared to LED A. Peak emission spectra are around $\sim 277 \mathrm{~nm}$ which are in the range of DUV LEDs [6]. A very minute blueshift is assigned to the reduction of quantum confinement stark effect in the proposed structure. 


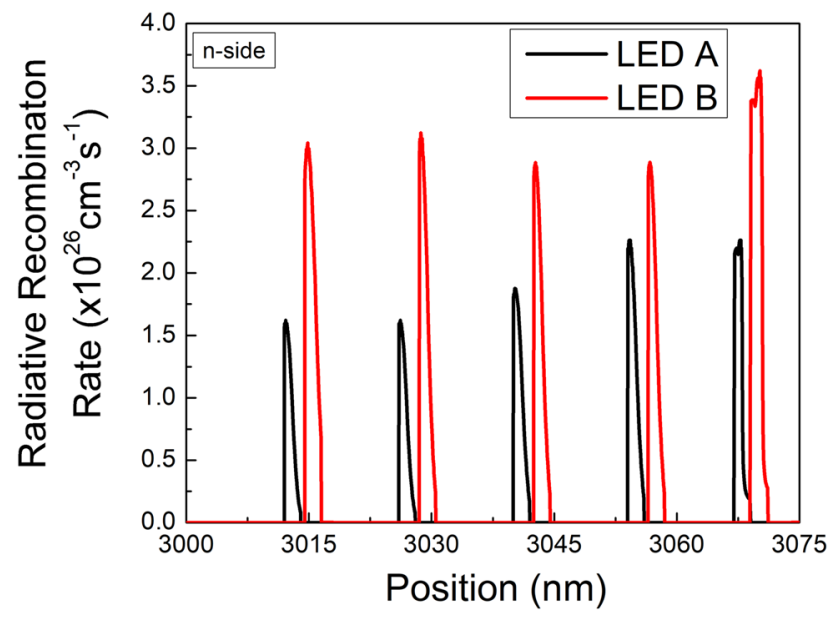

Fig. 5 Radiative recombination rates in the MQWs of both LEDs

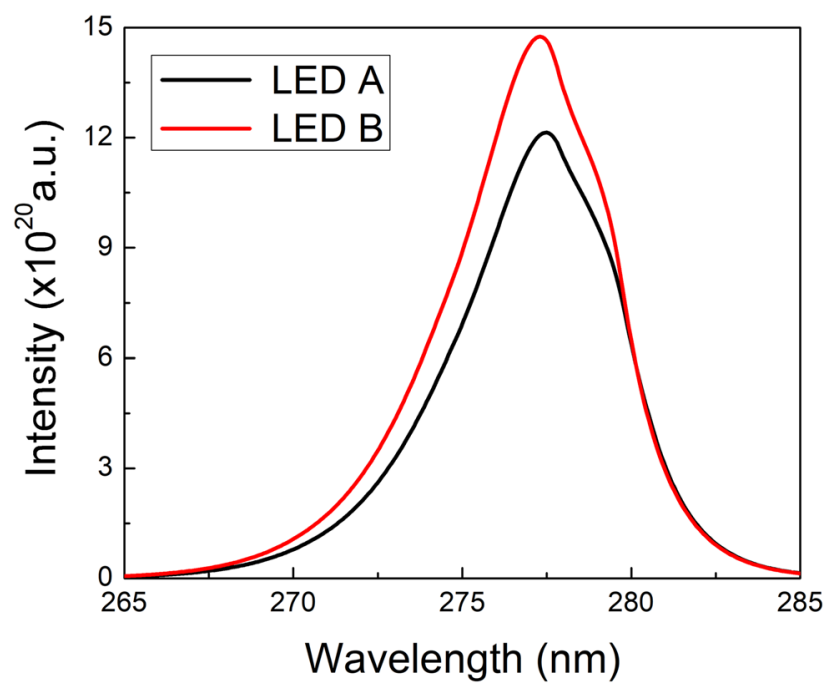

Fig. 6 Spontaneous emission spectrum for both LEDs
Figure 7 illustrates the band profile of both LEDs at $80 \mathrm{~A} /$ $\mathrm{cm}^{2}$. The $\Phi_{\mathrm{e}}$ and $\Phi_{\mathrm{h}}$ for electron and hole in LED A are $~ 334$ $\mathrm{meV}$ and $\sim 345 \mathrm{meV}$, respectively. Inversely, in LED-B the $\Phi_{\mathrm{e}}$ and $\Phi_{\mathrm{h}}$ are $\sim 443 \mathrm{meV}$ and $\sim 336 \mathrm{meV}$, respectively, indicating the better ability of electron confinement as well as hole transport into the MQWs region as shown in Fig. 7. It is due to the decrease of lattice mismatching leading to a lowering in polarization. It is highly believed that the above band-gap engineering is possible due to our proposed LED structure. In Fig. 3, 4, 5, and 7, the position (x-axis) is with reference to the device structure shown in Fig. 1a.

\section{Conclusion}

This study reported the effect of AlInGaN-based LQB and step-graded EBL on the optoelectronic behavior of AlGaNbased DUV LEDs. The results indicated that our approach improved the recombination of electron-hole pairs in the active region because of the elevation of potential barrier height of the conduction layer, which effectively suppressing the leakage of electrons. It is also attributed to the reduction of the induced polarization field, which is associated with lattice matching of epi-layers. Quantitively, the electron and hole concentrations are increased by $25 \%$ and $15 \%$ as compared to conventional LED. The IQE is enhanced by $48 \%$ while the efficiency droop is decreased from $52 \%$ to $3 \%$ in the proposed LED. So, not only the IQE is ameliorated but also the efficiency droop is stifled remarkably as compared to LED A. Therefore, we highly trust that this approach provides a feasible guideline to the researchers for achieving high IQE as well as near-droop-free efficient AlGaN-based DUV LEDs. 


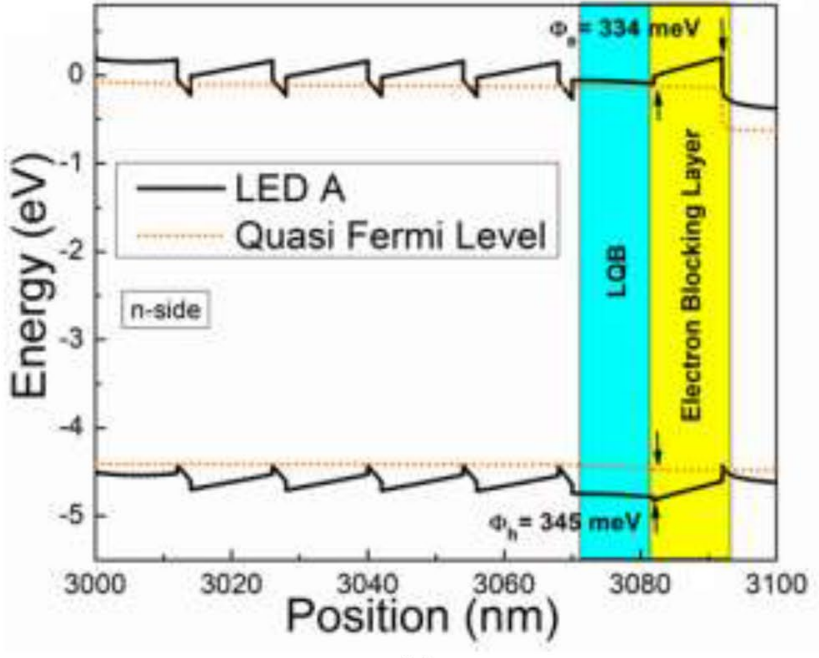

(a)

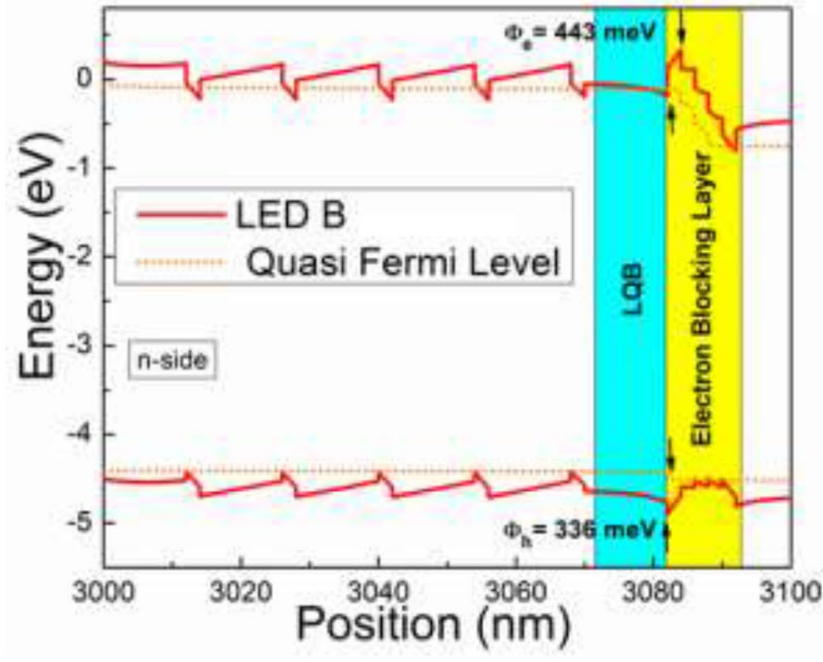

(b)

Fig. 7 Energy band profile a Conventional EBL structure (LED A), b LQB and step-graded EBL structure (LED B) at $80 \mathrm{~A} / \mathrm{cm}^{2}$

Acknowledgments The authors would like to thank Ghulam Ishaq Khan Institute of Engineering Sciences and Technology for providing the technical resources.

\section{Declarations}

Conflict of interest The authors declare no conflicts of interest.

\section{References}

1. H. Hirayama et al., Recent progress and future prospects of AlGaN-based high-efficiency deep-ultraviolet light-emitting diodes. Jpn. J. Appl. Phys. 53(10), 100209 (2014)

2. M. Würtele et al., Application of GaN-based ultraviolet-C light emitting diodes-UV LEDs-for water disinfection. Water Res. 45(3), 1481-1489 (2011)

3. K. Song, M. Mohseni, F. Taghipour, Application of ultraviolet light-emitting diodes (UV-LEDs) for water disinfection: a review. Water Res. 94, 341-349 (2016)

4. M.S Usman, M. Malik, M. Munsif, AlGaN-based ultraviolet lightemitting diodes: challenges and opportunities. Luminescence, (2020)

5. M. Buonanno et al., Far-UVC light $(222 \mathrm{~nm})$ efficiently and safely inactivates airborne human coronaviruses. Sci. Rep. 10(1), 1-8 (2020)

6. M. Kneissl, J. Rass, III-Nitride Ultraviolet Emitters (Springer, Cham, 2016).

7. E.F. Schubert, J.K. Kim, Solid-state light sources getting smart. Science 308(5726), 1274-1278 (2005)

8. M. Kneissl et al., The emergence and prospects of deep-ultraviolet light-emitting diode technologies. Nat. Photonics 13(4), 233-244 (2019)

9. F. Bouzid, L. Dehimi, F. Pezzimenti, Performance analysis of a Pt/n-GaN Schottky barrier UV detector. J. Electron. Mater. 46(11), 6563-6570 (2017)
10. J. Cho, E.F. Schubert, J.K. Kim, Efficiency droop in light-emitting diodes: challenges and countermeasures. Laser Photonics Rev. 7(3), 408-421 (2013)

11. H. Hirayama et al., 231-261 nm AlGaN deep-ultraviolet lightemitting diodes fabricated on AlN multilayer buffers grown by ammonia pulse-flow method on sapphire. Appl. Phys. Lett. 91(7), 071901 (2007)

12. J. Lang et al., High performance of AlGaN deep-ultraviolet light emitting diodes due to improved vertical carrier transport by deltaaccelerating quantum barriers. Appl. Phys. Lett. 114(17), 172105 (2019)

13. M. Usman et al., Improving AlGaN-based ultraviolet-C (UV-C) light-emitting diodes by introducing quaternary-graded AlInGaN final quantum barrier. Opt. Mater. 112, 110745 (2021)

14. S.M. Sze, Y. Li, K.K. Ng, Physics of Semiconductor Devices (Wiley, NewYork, 2021).

15. Y.-K. Kuo et al., Numerical investigation on the carrier transport characteristics of $\mathrm{AlGaN}$ deep-UV light-emitting diodes. IEEE J. Quantum Electron. 52(4), 1-5 (2016)

16. U. Muhammad, S. Malik, M.A. Khan, H. Hirayama, Suppressing the efficiency droop in the AlGaN-based UVB LED. Nanotechnology (2021)

17. A. Khan, K. Balakrishnan, T. Katona, Ultraviolet light-emitting diodes based on group three nitrides. Nat. Photonics 2(2), 77-84 (2008)

18. X. Fan et al., Efficiency improvements in AlGaN-based deep ultraviolet light-emitting diodes using inverted-V-shaped graded $\mathrm{Al}$ composition electron blocking layer. Superlattices Microstruct. 88, 467-473 (2015)

19. L. Wang et al., Enhanced optical performance of AlGaN-based deep-ultraviolet light-emitting diode with $\mathrm{m}$-shaped hole blocking layer and w-shaped electron blocking layer. Superlattices Microstruct. 133, 106188 (2019)

20. P. Qin et al., Improved performance of near UV light-emitting diodes with a composition-graded $\mathrm{p}$-AlGaN irregular sawtooth electron-blocking layer. Chin. Phys. B 25(8), 088505 (2016)

21. J. Li et al., Investigation of AlGaN-based near-ultraviolet lightemitting diodes with a trapezoidal electron blocking layer. J. Electron. Mater. 48(10), 6280-6286 (2019)

22. M. Usman, T. Jamil, S. Malik, H. Jamal, Designing anti-trapezoidal electron blocking layer for the amelioration of AlGaN-based 
deep ultraviolet light-emitting diodes internal quantum efficiency. Optik. 232, 166528 (2021)

23. Y. Liao et al., AlGaN based deep ultraviolet light emitting diodes with high internal quantum efficiency grown by molecular beam epitaxy. Appl. Phys. Lett. 98(8), 081110 (2011)

24. A. Kim et al., Performance of high-power AlInGaN light emitting diodes. Physica status solidi (a) 188(1), 15-21 (2001)

25. S.-J. Kim, K.J. Lee, S.-J. Park, Alleviation of efficiency droop in InGaN/GaN multiple quantum well light-emitting diodes with trapezoidal quantum barriers. J. Phys. D Appl. Phys. 51(25), 2501 (2018)

26. D. Demchenko, M. Reshchikov, Comment on "determination of the nitrogen vacancy as a shallow compensating center in GaN doped with divalent metals.” Phys. Rev. Lett. 115(2), 029701 (2015)

27. J. Wu, When group-III nitrides go infrared: new properties and perspectives. J. Appl. Phys. 106(1), 5 (2009)
28. N. Lobo et al., Enhancement of light extraction in ultraviolet lightemitting diodes using nanopixel contact design with $\mathrm{Al}$ reflector. Appl. Phys. Lett. 96(8), 081109 (2010)

29. I. Vurgaftman, J.A. Meyer, L.A. Ram-Mohan, Band parameters for III-V compound semiconductors and their alloys. J. Appl. Phys. 89(11), 5815-5875 (2001)

30. X. Chen, D. Wang, G. Fan, Investigation of AlGaN-based deepultraviolet light-emitting diodes with AlInGaN/AlInGaN superlattice electron blocking layer. J. Electron. Mater. 48(4), 2572-2576 (2019)

Publisher's Note Springer Nature remains neutral with regard to jurisdictional claims in published maps and institutional affiliations. 